\title{
DEVELOPMENT OF A BETA 0.12, 88 MHZ, QUARTER WAVE RESONATOR AND ITS CRYOMODULE FOR THE SPIRAL2 PROJECT
}

\author{
G. Olry, J-L. Biarrotte, S. Blivet, S. Bousson, C. Commeaux, C. Joly, T. Junquera, J. Lesrel, E. \\ Roy, H. Saugnac, P.Szott, CNRS/IN2P3/IPNO, Orsay, France
}

\section{Abstract}

SPIRAL2 is a radioactive beams facility, composed of a superconducting linac driver, delivering deuterons with an energy up to $40 \mathrm{MeV}(5 \mathrm{~mA})$ and heavy ions with an energy of $14.5 \mathrm{MeV} / \mathrm{u}(1 \mathrm{~mA})$. This facility is now fully approved by the French government.

The first prototype of beta 0.12 quarter-wave resonator has been recently fabricated by Zanon company and tested at IPN Orsay. The details on its fabrication and the results of the RF and mechanical tests at $4 \mathrm{~K}$ will be presented. Then, we will show the design of the cryomodule-B, dedicated to the high energy section of the linac, which is now ready to be ordered. Finally, the last studies of the R\&D program, such as the last optimizations of the geometry, the new developments of the tuning system and the design of the helium vessel, are described.

\section{BETA 0.12 QUARTER WAVE RESONATOR}

Details on RF and mechanical studies have already been presented in, respectively [1] and [2]. The goal is to reach an accelerating gradient of $6.5 \mathrm{MV} / \mathrm{m}$ with less than $10 \mathrm{~W}$ dissipated (NB: $\operatorname{Lacc}=\beta \lambda=0.41 \mathrm{~m})$.

\section{Fabrication}

The cavity was fabricated by Zanon SpA (Italy) within 6 months (Figure 1). The main problem came from the brazing of the $\varnothing 418 \mathrm{~mm}$ Stainless Steel ring (needed to fix the Helium vessel) which has required 3 tries.
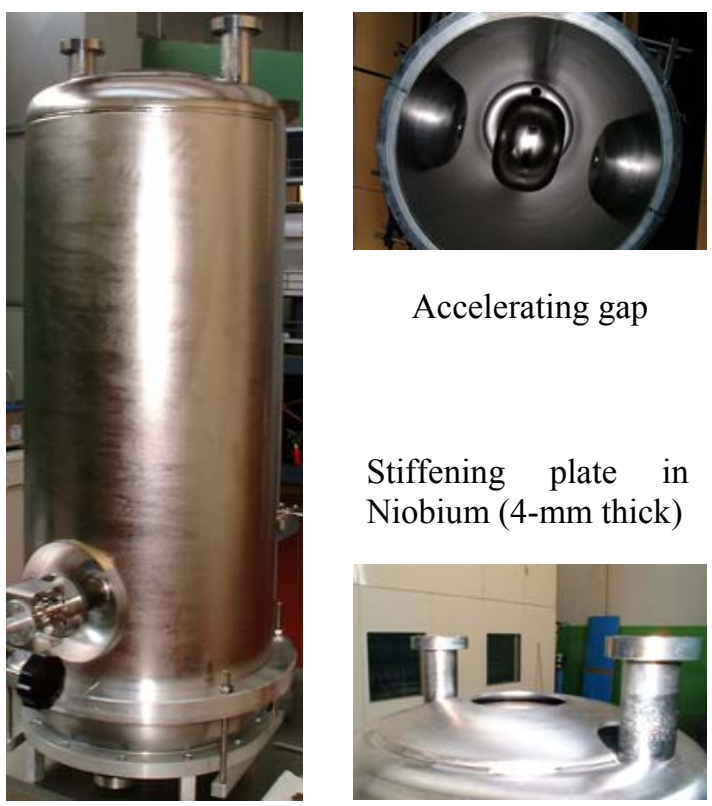

Accelerating gap

Stiffening plate in Niobium (4-mm thick)

Figure 1: Beta 0.12 QWR during the fabrication
RRR250 Niobium sheets from TokyoDenkaï were used: 4-mm thick for the cavity walls and 3-mm thick for the stem. The beam tubes and ports aperture is $36 \mathrm{~mm}$.

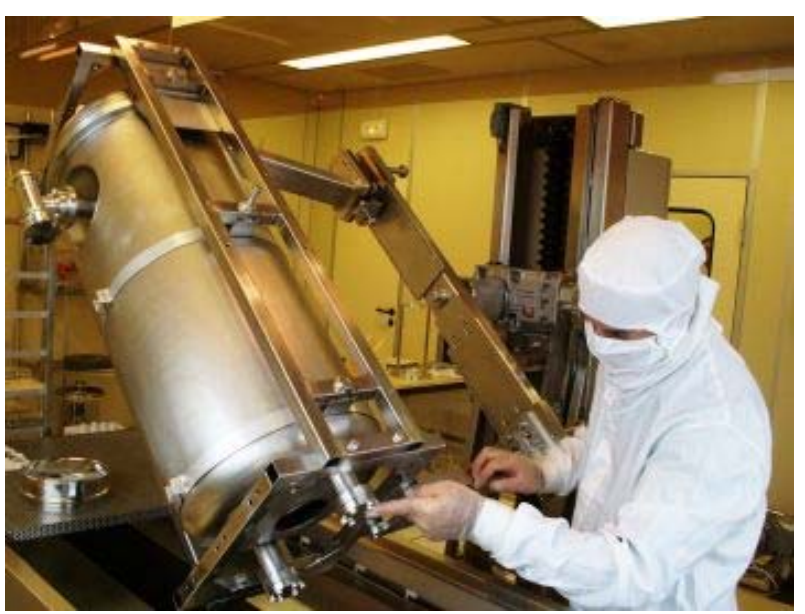

Figure 2: Beta 0.12 QWR inside the clean room before the High Pressure Rinsing process.

\section{Cold tests}

The preparation was done at CEA/Saclay: almost 200 $\mu \mathrm{m}$ were chemically removed (BCP), followed by a 2 hour rinsing process through the bottom and top ports (Figure 2).

A fixed capacitive coupler was used (Ø8 mm antenna) with a $1-\mathrm{kW}$ amplifier (limited to 250 Watts max). The cavity was tested 4 times between January and June (Figure 4) in our $\varnothing 800 \mathrm{~mm}$ vertical cryostat (Figure 3). Each test required 1500 litres of liquid Helium.

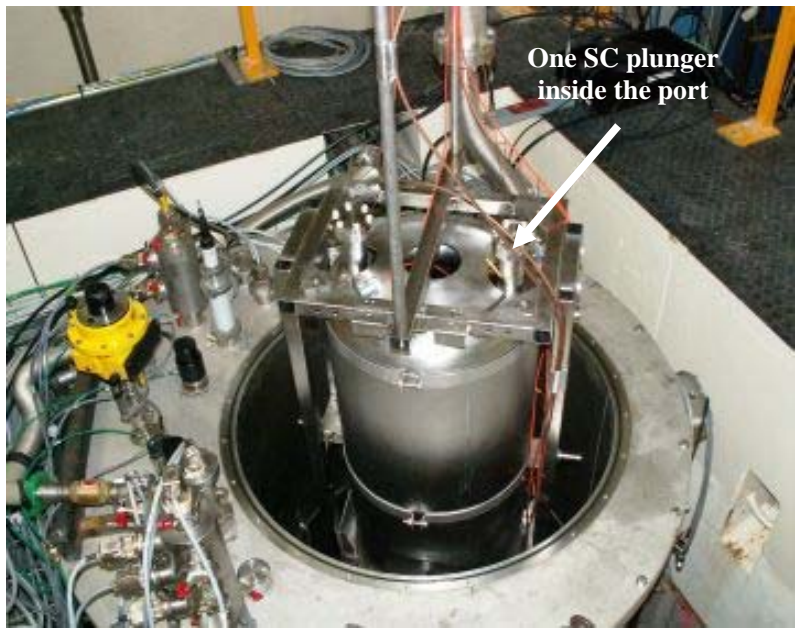

Figure 3: Beta 0.12 QWR before the test with a SC plunger in one of the top port. 


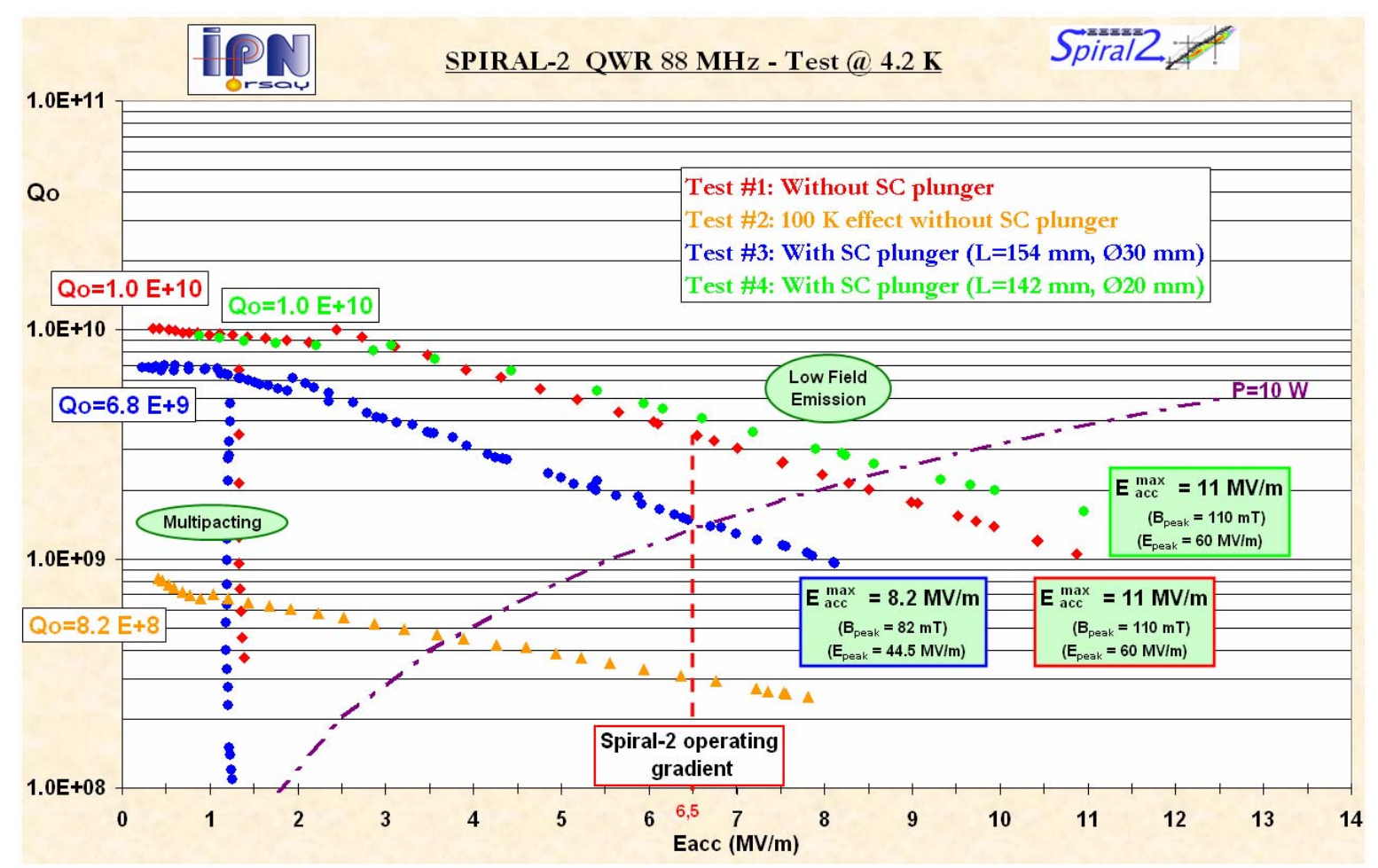

Figure 4: Cold tests results: Qo vs. Eacc

Both first experiments (see red and orange curves in Figure 4) were done without testing the static Niobium plungers (see $\S$ R\&D STUDIES for more details). We reached $11 \mathrm{MV} / \mathrm{m}$ (i.e. a voltage gain of $4.4 \mathrm{MV}$ ) for a dissipated power of $36 \mathrm{~W}$, without quenching. The Qo value at low field was $1.010^{10}$, corresponding to a residual resistance of $1.5 \mathrm{n} \Omega$. Then, after 5 days between $70 \mathrm{~K}$ and $130 \mathrm{~K}$, we observed the "100 K effect": i.e. Qo at low field $=8.210^{8}\left(\Leftrightarrow \mathrm{R}_{\text {residual }}=43\right.$ $\mathrm{n} \Omega)$.

During the last two experiments (see blue and green curves in Figure 4), we have tested two different Niobium plungers. The measured frequency shifts were: $+66.9 \mathrm{kHz}$ (test \#3) and $+18.2 \mathrm{kHz}$ (test \#4). 30 $\%$ of extra losses were measured using the $\varnothing 30 \mathrm{~mm}$ plunger: i.e. Qo $=6.810^{9}$ at low field. It was due to the magnetic field on the CF40 Stainless Steel flange (MAFIA simulations gave $20 \%$ of extra losses $\Leftrightarrow$ $\mathrm{Qo}=810^{9}$ ). For the last test, we used a shorter and thinner plunger. No Qo degradation was observed. We reached again $11 \mathrm{MV} / \mathrm{m}$ for $25 \mathrm{~W}$ dissipated inside the cavity without a quench.

One has to notice that we have faced, during each test, two multipacting barriers: a "strong" one (near 50 $\mathrm{kV} / \mathrm{m}$ !) which was processed in several hours and a "light" one, near $1.5 \mathrm{MV} / \mathrm{m}$, easily processed within a few minutes.

The static Lorentz detuning factor was measured: $\mathrm{K}_{\text {Lorentz meas. }}=-1.58 \mathrm{~Hz} /(\mathrm{MV} / \mathrm{m})^{2}$. The calculated value, by E. Zaplatin (FZJ), was $-1.52 \mathrm{~Hz} /(\mathrm{MV} / \mathrm{m})^{2}$, assuming an overall cavity thickness of $3.5 \mathrm{~mm}$.

\section{CRYOMODULE}

\section{Cryostat}

The two cavity helium tanks are fed by thermo siphon from a 20 litres helium buffer vessel installed inside the cryostat.

A thermal shield made of copper, is cooled between $60 \mathrm{~K}$ and $80 \mathrm{~K}$ with helium gas provided by the refrigerator. The cavity vacuum is separated from the cryostat insulation vacuum to prevent from pollution and allow the use of multilayer insulation inside the cryostat. The static cryogenic losses have been evaluated to be below $10 \mathrm{~W}$ at $4 \mathrm{~K}$ and below $65 \mathrm{~W}$ at $60 \mathrm{~K}$.

Cavities are individually maintained in position by mean of 4 horizontal and 3 vertical titanium alloy rods. In horizontal position, the $\mathrm{X}$ shaped antagonist rods keep the cavity position during cool down. The vertical displacement of the cavity is to be compensated by adjusting a misalignment during the assembly at $300 \mathrm{~K}$.

\section{Alignment procedures}

"Allen \& Robson" mires attached to the helium tank and precisely adjusted to the measured cavity axis materialize the real cavity axis. This axis can be measured through windows placed on the cryostat vacuum vessel. A third axis, materialized by "Allen \& Robson" mires, is then used to align all cryomodules with the different elements of the accelerators by mean of a laser tracker. 


\section{Assembly procedure}

The cryostat vacuum vessel and thermal shield are composed of several parts (Figure 5). Both cavities and their associated power coupler are mounted into clean room on the only central ring of the vacuum vessel (step 1 Figure 6). This assembly is done on a special support that performs a first rough alignment between the cavities axis and the cryostat axis. The other parts of the cryostat are assembled outside of the clean room as shown from step 2 to 7 on Figure 6.
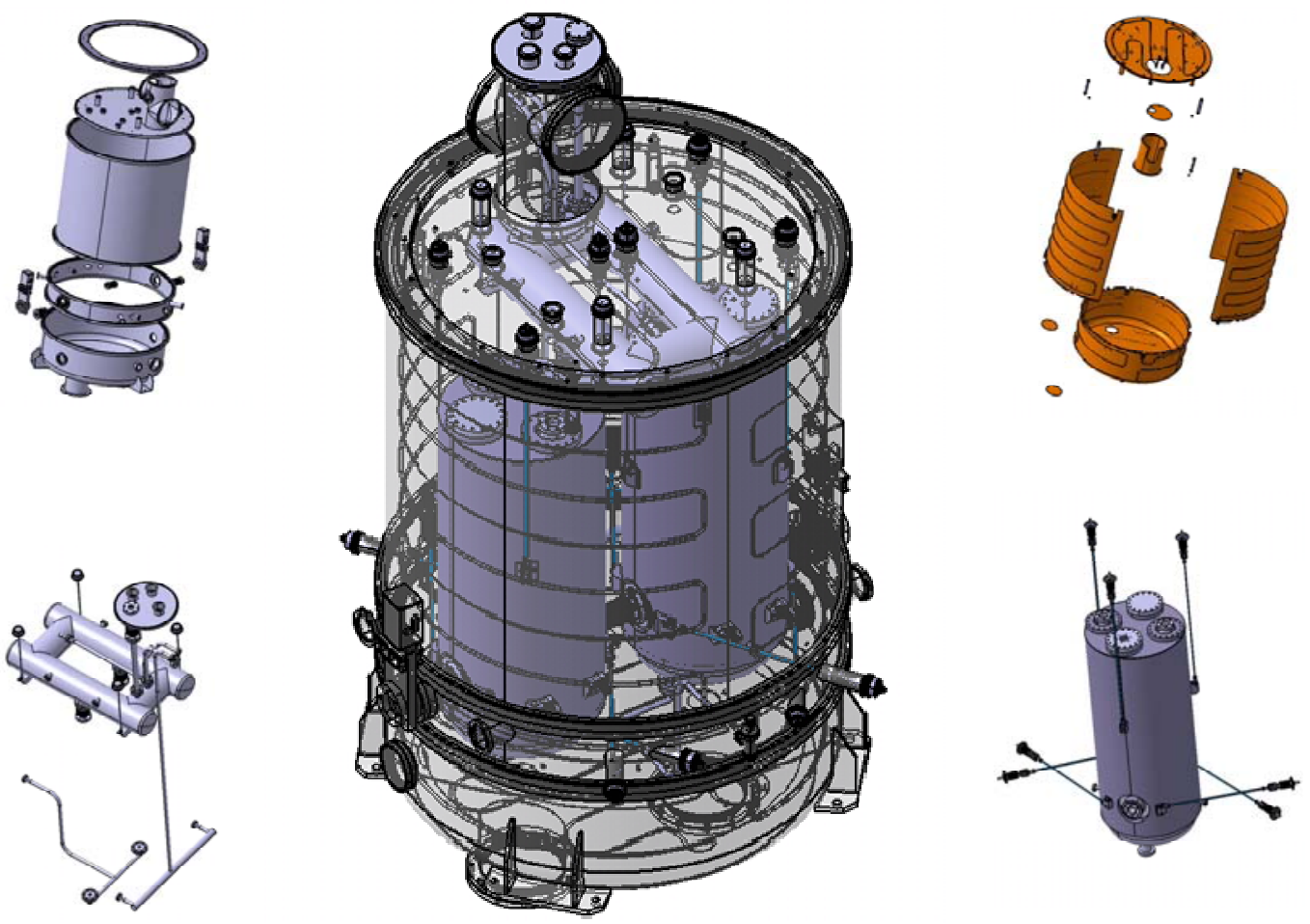

Figure 5: Beta 0.12 cryomodule
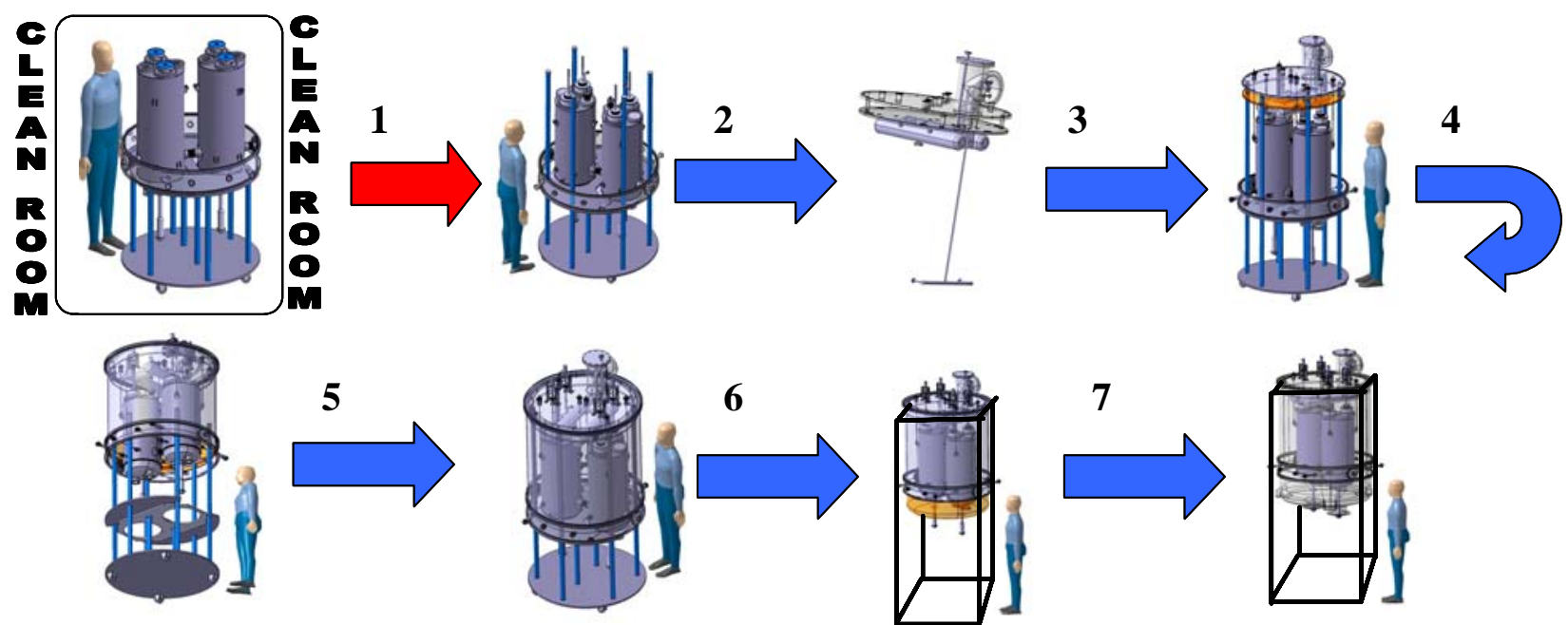

Figure 6: Cryostat assembly sequences 


\section{R\&D STUDIES}

\section{New cavity geometry}

We are now investigating a new shape of the drift tubes in order to avoid the "strong" multipacting barrier we observed at very low field level. We think the solution proposed for the modification of the halfwave resonator built by ACCEL could be a good solution [3]. We also look for rounded shape instead of titled walls.

\section{Cavity tuning systems}

A first warm tuning of the cavity was performed during the manufacturing of the first prototype. An adjustment of the gap distance within a range of $+/-2$ $\mathrm{mm}$ around the theoretical distance was done before the 3 last welds. An accuracy of $80 \mathrm{kHz}$ was achieved and should be improved for the next cavities.

Tuning by deforming the cavity walls is only possible by applying loads on the cylindrical body but gives a low sensitivity of around $4 \mathrm{kHz} / \mathrm{mm}$ and a tuning range of $8 \mathrm{kHz}$. A conceptual design of such a tuner is presented Figure 7.

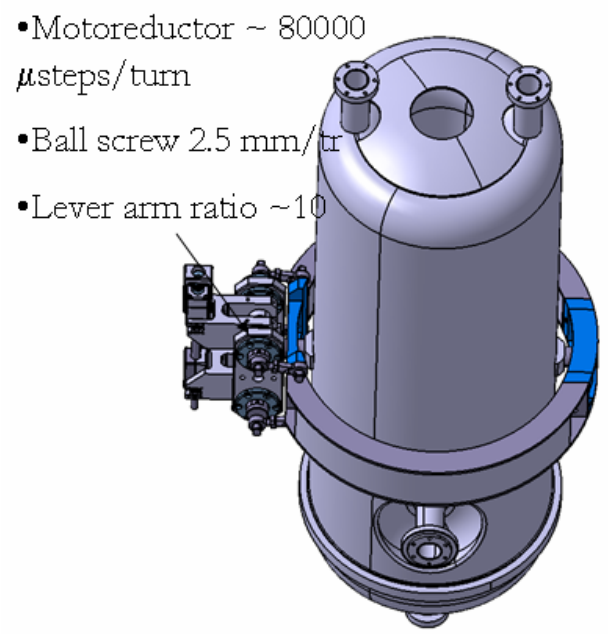

Figure 7: Cavity tuning system by deformation.

Due to the small tuning range of this solution, an alternative way to tune the cavity is investigated. It consists in introducing Niobium plungers cooled @ 4 $\mathrm{K}$ in the magnetic volume of the cavity. A first test on a rough prototype fixed on one of the upper cavity port shows encouraging results (see green curve in Figure 4) without any measurable effects on the RF cavity performances and a tuning shift of around $+18 \mathrm{kHz}$. A movable plunger is under study and is planned to be tested at the beginning of 2006. It is expected to obtain a sufficient dynamical adjustment of the frequency of $+/-4 \mathrm{kHz}$ around the nominal frequency, the cavity bandwidth being around $80 \mathrm{~Hz}$ at $4 \mathrm{~K}$.

The warm tuning will be performed by mixing the adjustment of the gap distance during manufacturing and the use of fixed plungers. For the dynamic cold tuning, tests are planed in 2006 to validate the best technical solution.

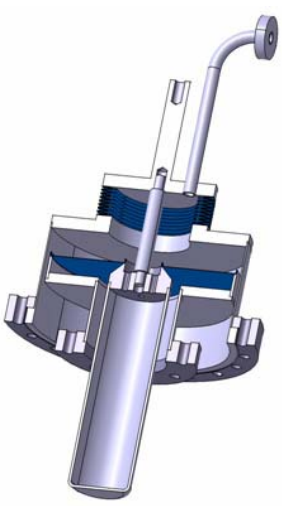

a- Movable

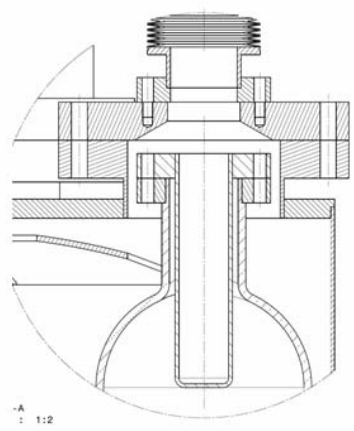

b-Static
Figure 8: SC plungers models.

\section{Helium vessel design}

The first prototype will have a Stainless Steel Helium vessel (currently under fabrication at Zanon $\mathrm{SpA}$, delivery foreseen in October). We are now designing a new vessel in Titanium (Figure 9) in order to avoid the brazing of the big Stainless Steel ring which has caused some problems and, more important, to fix the top of the stem on the vessel to compensate its vertical displacement and increase the frequency of the first mechanical vibration modes.

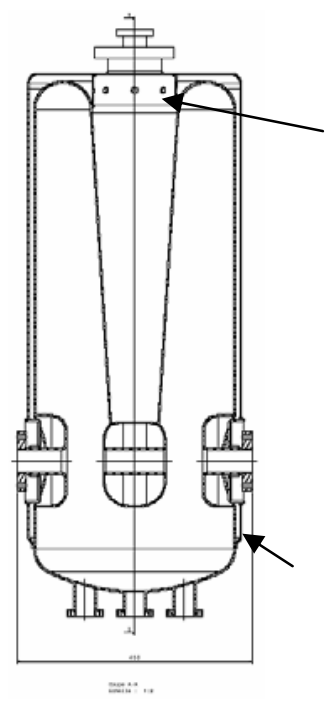

Connexion between the stem and the Helium vessel using a Titanium ring

Helium vessel welded on the cavity body

Figure 9: New design of the Helium vessel.

\section{CONCLUSION}

Following the good results obtained with the first prototype, we are going to launch the fabrication of two new cavities, equipped with their Titanium Helium tank and their dynamic cold tuning system. In parallel, the fabrication of the cryomodule is planned in 2006. The assembly will take place at IPN. The first test of the fully equipped cryomodule with two cavities and 
their power couplers is foreseen at the beginning of 2007.

\section{ACKNOWLEDGEMENTS}

The authors thank E. Zaplatin for his precious and precise calculations. We thank also the team of CEA/Saclay for their help during the preparation of the cavity.

\section{REFERENCES}

[1] T. Junquera et al., "High intensity linac driver for the SPIRAL 2 project", EPAC 2004, Lucerne, Switzerland, June 2004.

[2] H. Saugnac et al., "Mechanical stability simulations on a quarter wave resonator for the SPIRAL2 project", LINAC2004, Lübeck, Germany, August 2004.

[3] M. Pekeler et al., "Performance of a prototype 176 $\mathrm{MHz}$, beta 0.09 , half-wave resonator for the SARAF linac", these proceedings. 